\title{
Nutraceutical Interventions for Promoting Healthy Aging in Invertebrate Models
}

\author{
Yuqing Dong, ${ }^{1,2}$ Sujay Guha, ${ }^{1}$ Xiaoping Sun, ${ }^{3}$ Min Cao, ${ }^{1,2}$ Xiaoxia Wang, ${ }^{1}$ and Sige Zou ${ }^{3}$ \\ ${ }^{1}$ Department of Biological Sciences, Clemson University, 132 Long Hall, Clemson, SC 29634, USA \\ ${ }^{2}$ Institute for Engaged Aging, Clemson University, 535A Edwards Hall, Clemson, SC 29634, USA \\ ${ }^{3}$ Laboratory of Experimental Gerontology, National Institute on Aging, 251 Bayview Boulevard, Baltimore, MD 21224, USA \\ Correspondence should be addressed to Yuqing Dong, ydong@clemson.edu and Sige Zou, zous@grc.nia.nih.gov
}

Received 22 June 2012; Revised 8 August 2012; Accepted 10 August 2012

Academic Editor: Heinz D. Osiewacz

Copyright (๑) 2012 Yuqing Dong et al. This is an open access article distributed under the Creative Commons Attribution License, which permits unrestricted use, distribution, and reproduction in any medium, provided the original work is properly cited.

Aging is a complex and inevitable biological process that is associated with numerous chronically debilitating health effects. Development of effective interventions for promoting healthy aging is an active but challenging area of research. Mechanistic studies in various model organisms, noticeably two invertebrates, Caenorhabditis elegans and Drosophila melanogaster, have identified many genes and pathways as well as dietary interventions that modulate lifespan and healthspan. These studies have shed light on some of the mechanisms involved in aging processes and provide valuable guidance for developing efficacious aging interventions. Nutraceuticals made from various plants contain a significant amount of phytochemicals with diverse biological activities. Phytochemicals can modulate many signaling pathways that exert numerous health benefits, such as reducing cancer incidence and inflammation, and promoting healthy aging. In this paper, we outline the current progress in aging intervention studies using nutraceuticals from an evolutionary perspective in invertebrate models.

\section{Introduction}

Aging is associated with a gradual decline of physiological and cognitive functions [1]. Age is a major risk factor for numerous diseases, such as diabetes, cancer, and various degenerative diseases, including Alzheimer's, Huntington's, and Parkinson's diseases [2, 3]. A major challenge to health care systems around the world is how to encourage and maintain a healthy lifespan in large and increasing populations of elderly individuals [1]. Significant progress has been made over the past two decades in elucidating the molecular mechanisms of aging [4-7]. Hundreds of genetic factors, called longevity-related genes, have been identified to modulate lifespan and healthspan in model organisms ranging from yeast, worms, flies, and rodents. A number of the longevity-related genes fall into three conserved nutrient sensing pathways: target-of-rapamycin (TOR), insulin/IGF-1-like signaling (IIS), and sirtuin pathways [7-9]. These pathways primarily sense cellular amino acid, glucose, and $\mathrm{NAD}^{+}$or $\mathrm{NAD}^{+} / \mathrm{NADH}$ levels, respectively, (Figure 1). Genome-wide association studies have linked some longevity-related genes discovered in model organisms to human longevity, such as insulin-like growth factor receptor (IGF1R) and forkhead transcription factor (FOXO3a) in the IIS pathway [10-14]. Mechanistic studies of aging have led to the proposal of a number of hypotheses of aging. Perhaps the most prominent is the free radical hypothesis of aging, which states that free radicals, such as reactive oxygen species, generated from metabolism inflict oxidative damage to macromolecules, including protein, DNA, and lipid. Accumulation of such oxidative damage with age causes biological aging and eventually results in death $[15,16]$. However, numerous studies have now shown that this simplified version of free radical hypothesis of aging is not necessarily sufficient to explain the mechanisms underlying aging processes. Another is the hormesis hypothesis of aging, which has been frequently used to interpret the prolongevity effects induced by nutraceuticals. Hormesis theory states that mild stressinduced stimulation of defense response at the organismal level results in biologically beneficial effects and extends 


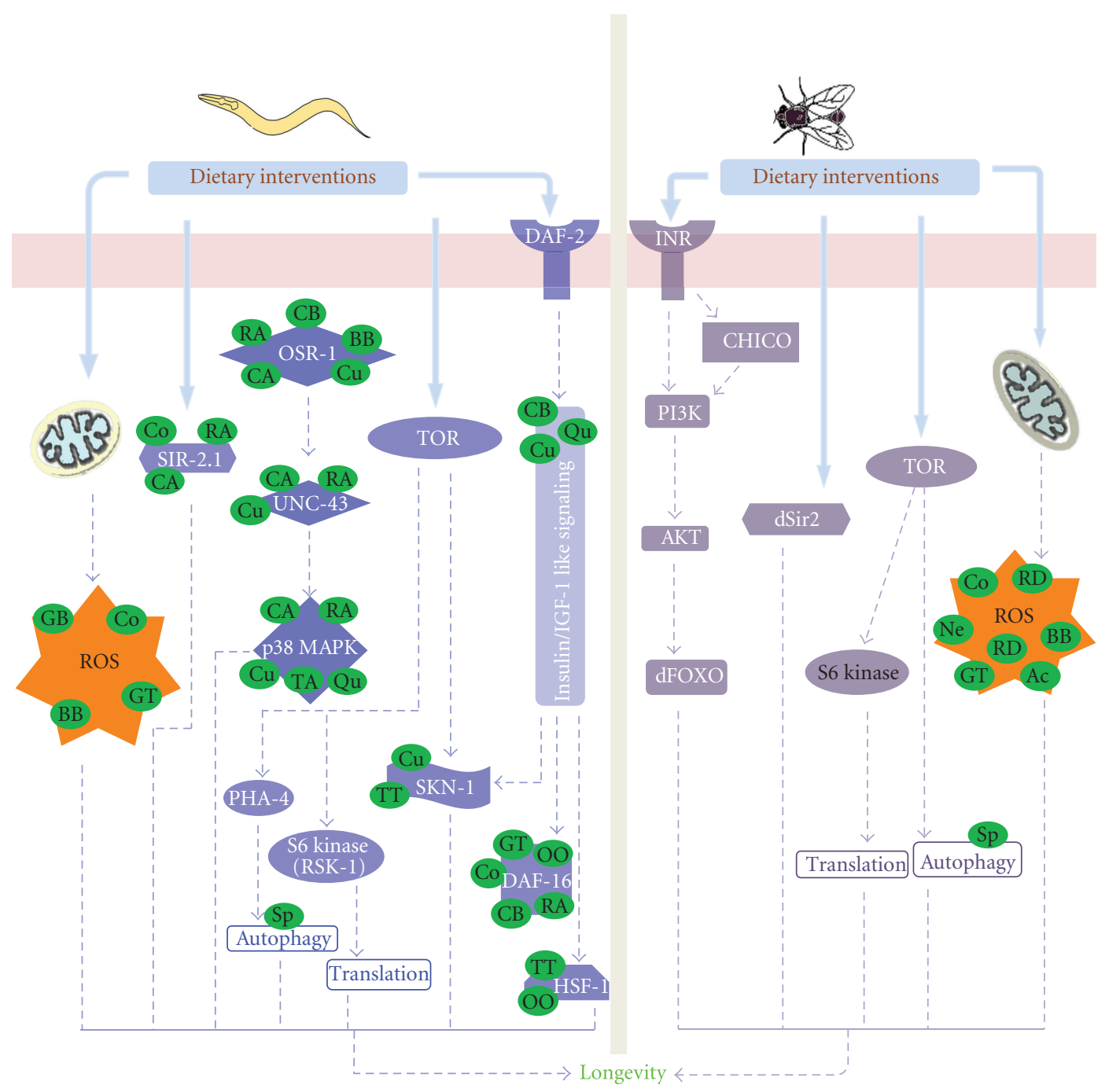

FIGURE 1: Nutrient sensing signaling and stress response pathways in C. elegans and D. melanogaster. Dietary interventions promote healthy lifespan and stress resistance through at least three conserved nutrient sensing pathways, IIS, TOR, and sirtuins. Many nutraceuticals improve healthspan by regulating ROS/redox state, some are known to interact with the IIS and osmotic resistant pathways to extend lifespan, and some modulate lifespan extension via autophagy mediated by TOR signaling. Each nutraceutical is represented by a green circle and the location of the green circle indicates the possible target of a nutraceutical action*. Ac: açai, BB: blueberry, CA: caffeic acid, CB: cranberry, Co: cocoa, Cu: curcumin, GB: ginkgo biloba, GT: green tea, Ne: nectarine, OO: olive oil, Qu: quercetin, RD: rosa damascene, RA: rosmarinic acid, Sp: spermidine, TA: tannic acid, and TT: thioflavin T. ${ }^{*}$ ROS presented in the figure indicates the regulation of ROS/redox state.

lifespan and healthspan. The defense response involves many protective mechanisms and influences with gene expression and metabolism [16-18]. Therefore, significant efforts in developing interventions for promoting healthy aging have been devoted to identify effective ways to modulate metabolism and stress [8].

Dietary restriction (DR) by modest reduction of all macronutrients or protein content is one of the most effective environmental interventions for promoting healthy aging [19-25]. However, it is challenging to implement dietary restriction in humans. A number of genes, including components in the conserved nutrient sensing pathways, have been identified to mediate lifespan extension induced by $\mathrm{DR}[9,26]$. As an alternative to DR, many studies have been devoted to the identification of pharmacological and nutraceutical reagents that extend lifespan and healthspan, some of which may mimic the effect of DR $[27,28]$. Due to their short lifespan and rich genetic resource, Drosophila melanogaster and Caenorhabditis elegans, have been in the forefront of research on aging interventions. In this paper, we focus on aging interventions in invertebrate models using nutraceuticals, which refer to extracts made out of edible fruits, vegetables, and herbs. Interventions with pharmacological reagents, such as resveratrol, metformin, and rapamycin, have been discussed in numerous comprehensive reviews elsewhere [28-33], and will not be described in this 
paper. Here we attempt to integrate the findings from studies on the mechanisms of aging and nutraceutical interventions with an evolutionary perspective.

\section{Dietary Botanicals and Phytochemicals}

Numerous studies have demonstrated the effects of nutraceuticals from fruit or plant extracts in reducing oxidative damage and promoting healthy aging in invertebrate models. The active ingredients in nutraceuticals that are generally produced by plants as "secondary compounds" appear to help plants overcome stressful conditions [34-36]. The beneficial properties of nutraceuticals can be attributed to the varieties of phytochemicals, such as flavonoids, anthocyanin glycosides, triterpenoids, and proanthocyanidin oligomers [37-39]. In this section, we will describe the antiaging properties of several nutraceuticals made from fruits, spices, and teas, which are commonly consumed by humans in daily life.

2.1. Ginkgo biloba Extract (EGb 761). Ginkgo is widely used as an ancient Chinese medicine due to its benefits to the health of the elderly [40]. The ginkgo biloba extract has been proposed to help prevent Alzheimer's disease (AD). The herbal extract EGb761 made from Ginkgo biloba is rich in flavonoids and terpenoids, which improves the functions of platelet and nerve cells and the blood flow to the nervous system and brain, probably due to its antioxidant properties [41, 42]. In wild type worms, EGb761 extends lifespan by $~ 10 \%$, reduces accumulation of oxidative damage, and represses expression of thermal stress-response protein hsp-16.2 [4346]. In addition, EGb 761 inhibits $A \beta$ oligomerization and alleviates $\mathrm{A} \beta$-induced paralysis in the $C$. elegans model of $\mathrm{AD}$ [47]. $\mathrm{A} \beta$ oligomerization is a hallmark of $\mathrm{AD}$ [48]. However, it remains to be determined whether ginkgo biloba can promote longevity in other models, such as Drosophila. Nevertheless, combined with studies demonstrating the neuroprotective function of EGb 761 in mammalian cells $[49,50]$, findings in worms suggest that the beneficial effect of EGb761 in prevention and treatment of $\mathrm{AD}$ could at least partially be due to its capacity to resist oxidative stress and reduce detrimental protein aggregation.

2.2. Blueberry. Blueberry contains a wide array of polyphenols and offers a host of health benefits [51]. Supplementation of blueberry preserves learning and memory in aged F344 rats by improving neuronal function [52]. Polyphenols from blueberry significantly increase the lifespan of C. elegans [53]. In C. elegans, blueberry supplementation decreases age-related accumulation of the intracellular level of lipofuscin, a biomarker for age-related cellular damage, and reduces the level of 4-Hydroxynonenal level, a biomarker for lipid peroxidation. In addition, blueberry polyphenols improve the pharyngeal pumping rates of the aged worms and increase thermotolerance, suggesting that blueberry improves worms' healthspan. Mechanistic studies indicate that blueberry supplementation reduces mRNA levels of heat shock proteins and requires OSR-1/UNC-43/SEK-1, components of the osmotic stress resistance pathway [53], to promote longevity in C. elegans. A recent study has demonstrated that blueberry extracts extends mean lifespan by approximately $10 \%$ in Drosophila [54]. Associated with lifespan extension, blueberry increased expression of several antioxidant genes, including superoxide dismutase 1 (SOD1), SOD2, and catalase (CAT) in Drosophila. Further genetic studies are needed to establish whether any of the antioxidant genes are required for lifespan extension induced by blueberry in Drosophila. These findings in invertebrates suggest that antioxidant machinery and/or osmotic stress pathway may play a pivotal and common role in mediating lifespan extension induced by blueberry.

2.3. Oregano and Cranberry. Cranberry and oregano possess multiple medicinal properties, such as antimicrobial, antiviral, antimutagenic, antiangiogenic, and antioxidation functions $[55,56]$. A mixture of oregano and cranberry (OC) extract increased lifespan in Mexican fruit flies (mexfly) in a diet composition dependent manner [57]. OC extended lifespan in mexflies fed diets with a relatively higher sugar to protein ratio, but not in mexflies fed diets with a lower sugar to protein ratio. OC supplementation did not compromise the lifetime reproductive output, a parameter of healthspan. In addition, OC supplementation in middle age was sufficient to promote longevity [58]. However, lifespan was not increased when OC was supplemented only in young age or old age. These findings point out the importance of considering diet composition and implementing time in developing an efficacious aging intervention.

We have recently assessed the effect of cranberry extract alone on lifespan and healthspan in C. elegans. Our data indicate that the cranberry extract alone is sufficient to prolong lifespan in C. elegans [59]. The cranberry-mediated lifespan extension was suppressed almost completely by the absence of DAF-16, a forkhead transcription factor in the IIS. Cranberry supplementation did not further extend the lifespan of DAF-2 or AGE-1 mutants, either. DAF-2 and AGE-1 are insulin-like receptor and phosphatidylinositol 3 kinase (PI3 K) in the IIS pathway of C. elegans [60, 61]. Cranberry is high in antioxidants and phytochemicals, including proanthocyanidins and vitamin $\mathrm{C}$, which may neutralize free radicals and reduce oxidative damage, and more importantly modulating signaling transduction pathways [51, 62]. Our findings suggest that IIS and DAF-16 play an important role in cranberry mediated prolongevity in C. elegans. Further studies are warranted to determine to what extent the prolongevity effect of cranberry attributes to its antioxidant properties.

2.4. Nectarine and Açai. Nectarine is a globally consumed fruit [63], and açai is a fruit indigenous to the Amazon River area [64]. Both fruits contain various kinds of bioactive phytochemicals $[2,3,65]$. Nectarine supplementation can extend lifespan in flies fed standard, DR, and high-fat diets. The lifespan extension induced by nectarine is associated with increased lifetime reproductive output and reduced lipid oxidation [66]. In contrast, supplementation of açai pulp promotes survival in Drosophila fed a high-fat but not a standard diet [67]. The diet composition dependent effect 
of açai is also evident in the mexfly. Açai supplementation promotes the survival of the mexfly fed a high-fat and highsugar diet but not other nonfat diets [68]. Along with the OC study described above, the importance of diet composition is also evident in aging intervention studies using pharmacological agents, such as resveratrol. Studies in Drosophila, mexfly, and mice have shown that the prolongevity effects of resveratrol depend on diet composition [69-71]. These studies again stress the importance of diet composition in modulating the health benefits of nutraceuticals. Moreover, both nectarine and açai can promote the survival of flies with sod 1 deficiency. sod 1 deficient flies have a short lifespan and experience high levels of oxidative damage. These findings suggest that nectarine and açai possess antioxidant activities at the organismal level $[26,67,72]$. However, it remains to be determined the mechanisms underlying the prosurvival effects of nectarine and açai.

2.5. Rosa damascena. A hybrid rose species, Rosa damascena, is traditionally used to make rose oil and water in cosmetic and food industries [73, 74]. Extracts from $R$. damascena contains numerous volatile organic compounds including various terpenes such as citronellol, heneicosane, and disiloxane, and also polyphenols, such as quercetin, myricetin, kaempferol, and gallic acid [75]. R. damascena extracts have been shown to possess biological properties that are protective against microbial infection, seizures in rats, and toxicity of amyloid beta in neurons, a biomarker of AD [7679]. An $R$. damascena extract has been found to increase both mean and maximum lifespan in Drosophila. This extract also enhances flies' resistance to oxidative stress and low iron stress, but paradoxically increases flies' sensitivity to heat stress. Molecular studies indicate that the rose extract reduces the heat-induced expression of a major heat shock protein HSP70 and a small mitochondrial heat shock protein HSP22 [80]. Therefore, it has been proposed that $R$. damascena extract extends lifespan by protecting flies against ironinduced stress.

2.6. Cocoa Polyphenols. Numerous polyphenols with high antioxidant activities, such as flavonoids, have been isolated from cocoa [81]. Flavonoid-enriched cocoa powder reduces oxidative stress in C. elegans [82]. Moreover, the flavonoidenriched cocoa powder also significantly increases the lifespan of wild type N2 worms [82]. This lifespan extension was dependent on both SIR-2.1 and DAF-16. In addition, the flavonoid-enriched cocoa powder failed to exert any longevity effect on daf-2 mutants, indicating that cocoa powder-mediated lifespan extension may also act through the IIS. Considering the mechanism by which SIR-2.1 and IIS modulates lifespan, cocoa polyphenols may promote longevity by reducing oxidative stress, influencing metabolism, and altering chromatin structure.

2.7. Green Tea. Green tea contains polyphenolic catechins that have been reported to have a number of health benefits, including prevention of $\mathrm{AD}$, Parkinson's disease, and heart disease [83]. Green tea can protect against angiogenesis and tumor formation [84]. The health benefits of green tea are mainly attributed to bioactive properties of its phytochemical constituents $[85,86]$. A number of polyphenolic catechins, including epicatechin (EC), epicatechin-3-gallate (ECG), epigallocatechin (EGC), epigallocatechin-3-gallate (EGCG), catechin, and gallocatechin (GC), are abundant in green tea [83]. Among these, EGCG is perhaps the most abundant catechin in green tea, and has been reported to induce antioxidant enzymes, including glutathione peroxidase, catalase, and glutathione $S$-transferase, in mice [87]. Similar results were obtained in worms and flies. Supplementation with green tea catechins increases the antioxidant enzymatic activity of superoxide dismutase and catalase in Drosophila [88, 89]. Green tea extends median lifespan by $36 \%$ and mean lifespan by $16 \%$ in flies [89]. In C. elegans, EGCG treatment significantly reduces ROS levels under both normal culture and oxidative stress conditions, and increases the expression of sod-3 and $h s p-16.2$ [90, 91]. Administration of EGCG significantly extends the lifespan of $C$. elegans under heat stress and oxidative stress $[90,91]$. In addition, a recent study demonstrated that L-theanine, a unique amino acid particularly enriched in green tea, promoted the survival of $C$. elegans in the presence of paraquat [92]. L-theanine has been reported to provide broad health benefits, such as antitumor, $\mathrm{AD}$ prevention, and blood pressure reduction [93-96]. Together, these findings suggest that green tea increases lifespan and stress resistance partially through its antioxidant properties.

2.8. Olive Oil. Numerous studies have demonstrated that consumption of olive oil has multiple beneficial effects on health and longevity in humans [97-101]. The abundance of phenolic compounds present in olive oil is considered to play an important role in exerting these healthy effects. Lastest studies have revealed that the tyrosol, one of the most abundant phenols in olive oil [102], significantly promotes the longevity of $C$. elegans, as well as the resistance to thermal and oxidative stress [103]. IIS/DAF-16 and HSF-1 are required in tyrosol-mediated prolongevity in C. elegans. hsp-12.6, a coregulated target gene of DAF-16, and HSF-1 [104], is significantly upregulated in adult worms after the tyrosol treatment. It has been reported that small heat shock proteins, including HSP-12.6, can extend lifespan and delay polyglutamine protein aggregation in C. elegans [104]. HSF1 is critical for maintaining protein homeostasis. Together, these studies suggest that tyrosol in olive oil extends lifespan by increasing oxidative stress resistance and thermotolerance as well as improving protein homeostasis [103].

2.9. Quercetin and Tannic Acid. Quercetin (3,3',4',5,7-pentahydroxyflavone) is one of the most important dietary flavonoids present in a wide array of foods. Numerous health benefits are linked to consumption of fruits and vegetables containing a high content of quercetin [105]. Quercetin has been shown to dramatically increase the lifespan of $C$. elegans [106-109]. However, the studies on the molecular basis yielded conflicting results. One study indicates that lifespan extension induced by quercetin supplementation requires DAF-2 and AGE-1, the components of C. elegans insulin/IGF signaling [106]. Quecertin induces nuclear translocation of 
DAF-16 [110]. This study suggests that the IIS pathway mediates the prolongevity effect of quercetin. However, this hypothesis has been challenged by another study, which indicates that quercetin mediated lifespan extension does not depend on DAF-16 [107]. Additional studies are warranted to resolve the conflicting observation and understand the mechanisms underlying the prolongevity effect of quercetin.

Tannic acid (TA) belongs to tannins, which are secondary metabolites of plants with many health effects. TA has the biological activities to prevent neurodegeneration [111], pathogen infection $[112,113]$, carcinogenesis $[114,115]$, and oxidative damage $[116,117]$. In addition, TA significantly increases the lifespan of worms [108]. The IIS pathway and DAF-16 are not essential for the prolongevity effects of TA. Only SEK-1, one component of the p38 MAPK pathway, has been shown to be essential for TA-induced lifespan extension.

Transcriptome studies indicate that quercetin affects expression of genes in the TGF- $\beta$ signaling, insulin-like signaling, and p38 MAPK pathways, while TA changes expressions of genes in the TGF- $\beta$ and the p38 MAPK pathways as well as the amino acid metabolism. Together, these studies suggest that TGF- $\beta$ and p38 MAPK pathways play crucial roles in mediating the prolongevity effects of quercetin and TA [118].

2.10. Caffeic Acid and Rosmarinic Acid. Caffeic acid (3-(3,4dihydroxyphenyl)-2-propenoic acid; CA) and Rosmarinic acid ( $\alpha$-o-caffeoyl-3, 4 -dihydroxyphenyl lactic acid; RA) are abundantly present in a variety of fruits, vegetables, and herbs. CA and RA have anticarcinogenesis, antioxidant, antimicrobial, anti-inflammatory, and antirheumatic properties [119-123]. CA and RA can prolong the healthy lifespan of C. elegans [124]. DAF-16, SIR-2.1, OSR-1, UNK-43, and SEK-1 are required for RA triggered lifespan extension. Similar results have been obtained for CA mediated lifespan extension except that DAF-16 does not appear to play a pivotal role in this lifespan extension. Together, these findings suggest that CA and RA promote lifespan extension through overlapping pathways involved in metabolism and stress response.

2.11. Spermidine. Spermidine is an important polyamine presented in citrus fruit and soybean, and has effects on epigenetic modifications, autophagy, and necrosis $[125,126]$. Polyamine concentrations and autophagy have been shown to decline in various organisms, including humans [127, 128]. Supplementation of spermidine prolongs the lifespan of C. elegans and Drosophila by $15 \%$ and $30 \%$, respectively [125]. A growing number of evidence shows that autophagy plays an essential role in the regulation of lifespan mediated by the TOR pathway [129-131]. Autophagy is required for spermidine-mediated lifespan extension in both worms and flies. It will be interesting to see if spermidine and its derivatives can confer lifespan extension in humans by, at least in part, enhancing the autophagy.

2.12. Curcumin and Thioflavin T. Curcumin (diferuloylmethane) is the pharmacologically active substance in turmeric
(Curcuma longa), and has been widely used as a herbal medicine in Asia. A large body of evidence indicates that curcumin possesses many biological activities, such as antioxidative, anti-inflammatory, anticancer, chemopreventive, and antineurodegenerative properties [132-136]. With its pleiotropic activities, curcumin has been considered as a potential aging intervention compound. Studies in Drosophila and C. elegans have demonstrated that curcumin can delay aging and prolong the lifespan $[137,138]$. Curcumin-treated flies exhibited enhanced resistance against to oxidative stress, improved locomotor activity, and higher tolerance to chemotherapy drugs. Curcumin reduces expression of Methuselah, a longevity gene, and genes in the IIS and JNK pathways. Curcumin-induced lifespan extension has been shown to be mediated by the OSR-1/UNC-43/SEK-1 pathway and possible the members of the IIS and SKN-1 pathways in C. elegans.

Protein homeostasis is an essential lifespan modulator of animals [139]. Dysfunctional protein homeostasis results in protein misfolding and aggregation, which is a hallmark of aging and age-related diseases [140]. ThT is a compound known to bind and inhibit aggregation of amyloids, which leads to various neurodegenerative disorders [141]. Flavonoid thioflavin T (4-(3,6-dimethyl-1,3-benzothiazole3-ium-2-yl)-N,N-dimethylaniline chloride, ThT) has been shown to promote longevity in C. elegans [142]. Treatment with $50 \mu \mathrm{M}$ ThT, a concentration conferring a largest increase of lifespan, significantly suppresses the protein aggregation mediated paralysis in worms. The prolongevity effect of ThT depends on HSF-1 and SKN-1, but independent of the IIS. Importantly, the beneficial effects of ThT require an intact machinery for maintaining protein homeostasis. Like ThT, curcumin also has the ability to bind and inhibit protein aggregates [142]. Treatment of both ThT and curcumin does not yield synergistic effects on longevity. ThT and curcumin may act through similar mechanisms, for example, improving protein homeostasis, to modulate aging and agerelated disorders.

\section{Concluding Remarks}

Nutraceuticals made from widely-consumed plant products represent both opportunities and challenges in aging interventions. On the one hand, nutraceuticals from plant extracts can promote longevity and improve healthspan through multiple mechanisms, such as reducing oxidative stress, altering signaling pathways, influencing metabolism, and maintaining protein homeostasis (Figure 1). Nutraceuticals that promote longevity in invertebrate models are often made from edible and relatively easily accessible fruits, vegetables, spices, and other plant products. Thus, when appropriately implemented nutraceutical interventions can be efficient and cost-effective ways for promoting healthy aging in humans.

On the other hand, major challenges remain to be addressed in implementing nutraceutical interventions. First, nutraceuticals contain numerous bioactive phytochemicals. It is a daunting task to determine which phytochemicals are the active ingredients for promoting health benefits, or determine how the beneficial effects are mediated through 
synergistic actions among multiple ingredients. Second, more research is needed to elucidate the molecular mechanisms underlying the actions of nutraceuticals, in order to better assess the effects of nutraceutical supplementation on the health of animals. For instance, more recent studies have revealed that ROS plays a physiologically vital role in signal transduction, gene regulation, and redox regulation [143]. The previous concept of the "free radical hypothesis of aging" has been modified and diversified ROS a messenger role in addition to its toxicity. Thus, it would be harmful to eliminate ROS completely. In the context, although many nutraceuticals have antioxidant properties, we need more careful exams to unravel the molecular basis rather than simply attribute to their ability to scavenge free radicals. It is in line with this notion that some nutraceuticals, such as quercetin and blueberry polyphenols, modulate lifespan by activating molecular pathways independent to their direct antioxidant capacity $[53,107]$. Third, it is essential to investigate the impact of both short-term and long-term nutraceutical interventions on healthspan. A recently developed behavioral monitoring system capable of recording lifetime behavioral changes at a high resolution represents an example of a useful tool for assessing healthspan in invertebrates [144]. Lastly, an important and challenging issue is to further understand how the effects of nutraceuticals depend on diet composition and genetic background. The prolongevity effects of several nutraceuticals have been shown to depend on the timing of supplementation and the composition of diet [143, 145-148]. The diet composition-dependent effects will have a significant impact on the increasing demand for personalized nutritional intervention. The individual and synergistic effects of nutraceuticals as a component of dietary composition will require further study and scientific scrutiny, particularly since little regulatory oversight regarding their sale for human consumption currently exists.

We have highlighted how nutraceuticals prevent and protect against aging and stress in invertebrate models. Some of nutraceuticals and their synthetic derivatives are being tested for their therapeutic potential [149]. Numerous promising results have been obtained in model organisms that suggest evolutionarily conserved mechanisms are involved in their beneficial effects. Much progress has been made to decipher the molecular mechanisms of aging shared among multiple species, which provide valuable guidance for aging interventions. However, further extensive studies will be required to demonstrate whether any nutraceuticals or pharmaceuticals can effectively delay aging or age-related disease in humans. A wider range of additional assays should be considered to help us better understand aging processes and improve the quality and quantity of human life in the foreseeable future.

\section{Acknowledgments}

The authors thank Edward Spangler and Robert Kosinski for critical reading of the paper. They also thank Cole Murbach for editorial assistance. This work was supported by the Creative Inquiry fund at Clemson University and the Yamada Research Grant to Y. Dong and M. Cao, an American
Federation of Aging Research (AFAR) grant to Y. Dong and the Intramural Research Program at the National Institute on Aging, NIH to S. Zou.

\section{References}

[1] E. De Luca d'Alessandro, S. Bonacci, and G. Giraldi, "Aging populations: the health and quality of life of the elderly," La Clinica Terapeutica, vol. 162, no. 1, pp. e13-e18, 2011.

[2] V. Pizza, A. Agresta, C. W. ĎAcunto, M. Festa, and A. Capasso, "Neuroinflamm-aging and neurodegenerative diseases: an overview," CNS and Neurological Disorders, vol. 10, no. 5, pp. 621-634, 2011.

[3] L. Fontana, "Modulating human aging and age-associated diseases," Biochimica et Biophysica Acta, vol. 1790, no. 10, pp. 1133-1138, 2009.

[4] F. Peysselon and S. Ricard-Blum, "Understanding the biology of aging with interaction networks," Maturitas, vol. 69, no. 2, pp. 126-130, 2011.

[5] G. M. Martin, "The biology of aging: 1985-2010 and beyond," The FASEB Journal, vol. 25, no. 11, pp. 3756-3762, 2011.

[6] C. J. Kenyon, "The genetics of ageing," Nature, vol. 464, no. 7288, pp. 504-512, 2010.

[7] L. Fontana, L. Partridge, and V. D. Longo, "Extending healthy life span-from yeast to humans," Science, vol. 328, no. 5976, pp. 321-326, 2010.

[8] M. C. Haigis and B. A. Yankner, "The aging stress response," Molecular Cell, vol. 40, no. 2, pp. 333-344, 2010.

[9] N. Alic and L. Partridge, "Death and dessert: nutrient signalling pathways and ageing," Current Opinion in Cell Biology, vol. 23, no. 6, pp. 738-743, 2011.

[10] E. Ziv and D. Hu, "Genetic variation in insulin/IGF-1 signaling pathways and longevity," Ageing Research Reviews, vol. 10, no. 2, pp. 201-204, 2011.

[11] M. Soerensen, S. Dato, K. Christensen et al., "Replication of an association of variation in the FOXO3A gene with human longevity using both case-control and longitudinal data," Aging Cell, vol. 9, no. 6, pp. 1010-1017, 2010.

[12] L. Pawlikowska, D. Hu, S. Huntsman et al., "Association of common genetic variation in the insulin/IGF1 signaling pathway with human longevity," Aging Cell, vol. 8, no. 4, pp. 460-472, 2009.

[13] F. Flachsbart, A. Caliebe, R. Kleindorp et al., "Association of FOXO3A variation with human longevity confirmed in German centenarians," Proceedings of the National Academy of Sciences of the United States of America, vol. 106, no. 8, pp. 2700-2705, 2009.

[14] B. J. Willcox, T. A. Donlon, Q. He et al., "FOXO3A genotype is strongly associated with human longevity," Proceedings of the National Academy of Sciences of the United States of America, vol. 105, no. 37, pp. 13987-13992, 2008.

[15] D. Harman, "Aging: a theory based on free radical and radiation chemistry," Journal of Gerontology, vol. 11, no. 3, pp. 298-300, 1956.

[16] A. B. Salmon, A. Richardson, and V. I. Pérez, "Update on the oxidative stress theory of aging: does oxidative stress play a role in aging or healthy aging?" Free Radical Biology and Medicine, vol. 48, no. 5, pp. 642-655, 2010.

[17] M. Ristow and K. Zarse, "How increased oxidative stress promotes longevity and metabolic health: the concept of mitochondrial hormesis (mitohormesis)," Experimental Gerontology, vol. 45, no. 6, pp. 410-418, 2010. 
[18] S. I. S. Rattan, H. Sejersen, R. A. Fernandes, and W. Luo, "Stress-mediated hormetic modulation of aging, wound healing, and angiogenesis in human cells," Annals of the New York Academy of Sciences, vol. 1119, no. 1, pp. 112-121, 2007.

[19] C. M. McCay, M. F. Crowell, and L. A. Maynard, "The effect of retarded growth upon the length of life span and upon the ultimate body size," Journal of Nutrition, vol. 10, pp. 63-79, 1935.

[20] R. Weindruch, R. L. Walford, S. Fligiel, and D. Guthrie, "The retardation of aging in mice by dietary restriction: longevity, cancer, immunity and lifetime energy intake," Journal of Nutrition, vol. 116, no. 4, pp. 641-654, 1986.

[21] J. C. Jiang, E. Jaruga, M. V. Repnevskaya, and S. M. Jazwinski, "An intervention resembling caloric restriction prolongs life span and retards aging in yeast," The FASEB Journal, vol. 14, no. 14, pp. 2135-2137, 2000.

[22] D. J. Clancy, D. Gems, E. Hafen, S. J. Leevers, and L. Partridge, "Dietary restriction in long-lived dwarf flies," Science, vol. 296, no. 5566, p. 319, 2002.

[23] W. Mair and A. Dillin, "Aging and survival: the genetics of life span extension by dietary restriction," Annual Review of Biochemistry, vol. 77, pp. 727-754, 2008.

[24] R. J. Colman, R. M. Anderson, S. C. Johnson et al., "Caloric restriction delays disease onset and mortality in rhesus monkeys," Science, vol. 325, no. 5937, pp. 201-204, 2009.

[25] E. L. Greer and A. Brunet, "Different dietary restriction regimens extend lifespan by both independent and overlapping genetic pathways in C. elegans," Aging Cell, vol. 8, no. 2, pp. 113-127, 2009.

[26] M. D. W. Piper and A. Bartke, "Diet and aging," Cell Metabolism, vol. 8, no. 2, pp. 99-104, 2008.

[27] E. Morselli, M. C. Maiuri, M. Markaki et al., "Caloric restriction and resveratrol promote longevity through the Sirtuin1-dependent induction of autophagy," Cell Death and Disease, vol. 1, no. 1, article e10, 2010.

[28] L. Mouchiroud, L. Molin, N. Dallière, and F. Solari, "Life span extension by resveratrol, rapamycin, and metformin: the promise of dietary restriction mimetics for an healthy aging," BioFactors, vol. 36, no. 5, pp. 377-382, 2010.

[29] A. R. Mendelsohn and J. W. Larrick, "Rapamycin as an antiaging therapeutic? Targeting mammalian target of rapamycin to treat Hutchinson-Gilford progeria and neurodegenerative diseases," Rejuvenation Research, vol. 14, no. 4, pp. 437-441, 2011.

[30] G. E. Mullin, "Red wine, grapes, and better health-resveratrol," Nutrition in Clinical Practice, vol. 26, no. 6, pp. 722-723, 2011.

[31] O. Vang, N. Ahmad, C. A. Baile et al., "What is new for an old molecule? systematic review and recommendations on the use of resveratrol," PLoS ONE, vol. 6, no. 6, Article ID e19881, 2011.

[32] S. Bulterijs, "Metformin as a geroprotector," Rejuvenation Research, vol. 14, no. 5, pp. 469-482, 2011.

[33] F. Madeo, T. Eisenberg, S. Büttner, C. Ruckenstuhl, and G. Kroemer, "Spermidine: a novel autophagy inducer and longevity elixir," Autophagy, vol. 6, no. 1, pp. 160-162, 2010.

[34] B. Winkel-Shirley, "Biosynthesis of flavonoids and effects of stress," Current Opinion in Plant Biology, vol. 5, no. 3, pp. 218-223, 2002.

[35] P. Reymond, H. Weber, M. Damond, and E. E. Farmer, "Differential gene expression in response to mechanical wounding and insect feeding in Arabidopsis," Plant Cell, vol. 12, no. 5, pp. 707-719, 2000.
[36] D. Hermsmeier, U. Schittko, and I. T. Baldwin, "Molecular interactions between the specialist herbivore Manduca sexta (Lepidoptera, Sphingidae) and its natural host Nicotiana attenuata. I. Large-scale changes in the accumulation of growth- and defense-related plant mRNAs," Plant Physiology, vol. 125, no. 2, pp. 683-700, 2001.

[37] R. Tsao, "Chemistry and biochemistry of dietary polyphenols," Nutrients, vol. 2, no. 12, pp. 1231-1246, 2010.

[38] D. O. Kennedy and E. L. Wightman, "Herbal extracts and phytochemicals: plant secondary metabolites and the enhancement of human brain function," Advances in Nutrition, vol. 2, no. 1, pp. 32-50, 2011.

[39] A. Salminen, A. Kauppinen, and K. Kaarniranta, "Phytochemicals suppress nuclear factor-kappaB signaling: impact on health span and the aging process," Current Opinion in Clinical Nutrition \& Metabolic Care, vol. 15, no. 1, pp. 23-28, 2012.

[40] T. Y. Wu, C. P. Chen, and T. R. Jinn, "Traditional Chinese medicines and Alzheimer's disease," Taiwanese Journal of Obstetrics and Gynecology, vol. 50, no. 2, pp. 131-135, 2011.

[41] B. A. Abdel-Wahab and S. M. Abd El-Aziz, "Ginkgo biloba protects against intermittent hypoxia-induced memory deficits and hippocampal DNA damage in rats," Phytomedicine, vol. 19, no. 5, pp. 444-450, 2012.

[42] F. V. DeFeudis and K. Drieu, "Ginkgo biloba extract (EGb 761) and CNS functions: basic studies and clinical applications," Current Drug Targets, vol. 1, no. 1, pp. 25-58, 2000.

[43] A. Kampkötter, T. Pielarski, R. Rohrig et al., "The Ginkgo biloba extract EGb761 reduces stress sensitivity, ROS accumulation and expression of catalase and glutathione Stransferase 4 in Caenorhabditis elegans," Pharmacological Research, vol. 55, no. 2, pp. 139-147, 2007.

[44] J. Vining Smith and Y. Luo, "Elevation of oxidative free radicals in Alzheimer's disease models can be attenuated by Ginkgo biloba extract EGb 761," Journal of Alzheimer's Disease, vol. 5, no. 4, pp. 287-300, 2003.

[45] A. Strayer, Z. Wu, Y. Christen, C. D. Link, and Y. Luo, "Expression of the small heat-shock protein Hsp16-2 in Caenorhabditis elegans is suppressed by Ginkgo biloba extract EGb 761," The FASEB Journal, vol. 17, no. 15, pp. 2305-2307, 2003.

[46] Z. Wu, J. V. Smith, V. Paramasivam et al., "Ginkgo biloba extract EGb 761 increases stress resistance and extends life span of Caenorhabditis elegans," Cellular and Molecular Biology, vol. 48, no. 6, pp. 725-731, 2002.

[47] Y. Wu, Z. Wu, P. Butko et al., "Amyloid- $\beta$-induced pathological behaviors are suppressed by Ginkgo biloba extract EGB 761 and ginkgolides in transgenic Caenorhabditis elegans," Journal of Neuroscience, vol. 26, no. 50, pp. 13102-13113, 2006.

[48] V. H. Finder and R. Glockshuber, "Amyloid- $\beta$ aggregation," Neurodegenerative Diseases, vol. 4, no. 1, pp. 13-27, 2007.

[49] Y. Luo, J. V. Smith, V. Paramasivam et al., "Inhibition of amyloid- $\beta$ aggregation and caspase- 3 activation by the Ginkgo biloba extract EGb761," Proceedings of the National Academy of Sciences of the United States of America, vol. 99, no. 19, pp. 12197-12202, 2002.

[50] J. V. Smith, A. J. Burdick, P. Golik, I. Khan, D. Wallace, and Y. Luo, "Anti-apoptotic properties of Ginkgo biloba extract EGb 761 in differentiated PC12 cells," Cellular and Molecular Biology, vol. 48, no. 6, pp. 699-707, 2002.

[51] C. C. Neto, "Cranberry and blueberry: evidence for protective effects against cancer and vascular diseases," Molecular 
Nutrition and Food Research, vol. 51, no. 6, pp. 652-664, 2007.

[52] J. A. Joseph, B. Shukitt-Hale, N. A. Denisova et al., "Reversals of age-related declines in neuronal signal transduction, cognitive, and motor behavioral deficits with blueberry, spinach, or strawberry dietary supplementation," Journal of Neuroscience, vol. 19, no. 18, pp. 8114-8121, 1999.

[53] M. A. Wilson, B. Shukitt-Hale, W. Kalt, D. K. Ingram, J. A. Joseph, and C. A. Wolkow, "Blueberry polyphenols increase lifespan and thermotolerance in Caenorhabditis elegans," Aging Cell, vol. 5, no. 1, pp. 59-68, 2006.

[54] C. Peng, Y. Zuo, K. M. Kwan et al., "Blueberry extract prolongs lifespan of Drosophila melanogaster," Experimental Gerontology, vol. 47, no. 2, pp. 170-178, 2012.

[55] E. Pappas and K. M. Schaich, "Phytochemicals of cranberries and cranberry products: characterization, potential health effects, and processing stability," Critical Reviews in Food Science and Nutrition, vol. 49, no. 9, pp. 741-781, 2009.

[56] F. E. Babili, J. Bouajila, J. P. Souchard et al., "Oregano: chemical analysis and evaluation of its antimalarial, antioxidant, and cytotoxic activities," Journal of Food Science, vol. 76, no. 3, pp. C512-C518, 2011.

[57] S. Zou, J. R. Carey, P. Liedo, D. K. Ingram, B. Yu, and R. Ghaedian, "Prolongevity effects of an oregano and cranberry extract are diet dependent in the mexican fruit fly (Anastrepha ludens)," Journals of Gerontology, vol. 65, no. 1, pp. 4150, 2010.

[58] S. Zou, J. R. Carey, P. Liedo, D. K. Ingram, and B. Yu, "Prolongevity effects of a botanical with oregano and cranberry extracts in Mexican fruit flies: examining interactions of diet restriction and age," Age, vol. 34, no. 2, pp. 269-279, 2012.

[59] S. Guha, M. Cao, R. M. Kane, A. M. Savino, S. Zou, and Y. Dong, "The longevity effect of cranberry extract in Caenorhabditis elegans is modulated by daf-16 and osr-1," Age. In press.

[60] E. Cohen and A. Dillin, "The insulin paradox: aging, proteotoxicity and neurodegeneration," Nature Reviews Neuroscience, vol. 9, no. 10, pp. 759-767, 2008.

[61] J. Z. Morris, H. A. Tissenbaum, and G. Ruvkun, "A phosphatidylinositol-3-OH kinase family member regulating longevity and diapause in Caenorhabditis elegans," Nature, vol. 382, no. 6591, pp. 536-539, 1996.

[62] X. He and H. L. Rui, "Cranberry phytochemicals: isolation, structure elucidation, and their antiproliferative and antioxidant activities," Journal of Agricultural and Food Chemistry, vol. 54, no. 19, pp. 7069-7074, 2006.

[63] D. Konopacka, K. Jesionkowska, D. Kruczyńska et al., "Apple and peach consumption habits across European countries," Appetite, vol. 55, no. 3, pp. 478-483, 2010.

[64] A. G. Schauss, X. Wu, R. L. Prior et al., "Phytochemical and nutrient composition of the freeze-dried amazonian palm berry, Euterpe oleraceae Mart. (Acai)," Journal of Agricultural and Food Chemistry, vol. 54, no. 22, pp. 8598-8603, 2006.

[65] A. G. Schauss, X. Wu, R. L. Prior et al., "Antioxidant capacity and other bioactivities of the freeze-dried Amazonian palm berry, Euterpe oleraceae Mart. (Acai)," Journal of Agricultural and Food Chemistry, vol. 54, no. 22, pp. 8604-8610, 2006.

[66] O. Boyd, P. Weng, X. Sun et al., "Nectarine promotes longevity in Drosophila melanogaster," Free Radical Biology and Medicine, vol. 50, no. 11, pp. 1669-1678, 2011.

[67] X. Sun, J. Seeberger, T. Alberico et al., "Açai palm fruit (Euterpe oleracea Mart.) pulp improves survival of flies on a high fat diet," Experimental Gerontology, vol. 45, no. 3, pp. 243-251, 2010.
[68] P. Liedo, J. R. Carey, D. K. Ingram, and S. Zou, "The interplay among dietary fat, sugar, protein and acai (Euterpe oleracea Mart.) pulp in modulating lifespan and reproduction in a Tephritid fruit fly," Experimental Gerontology, vol. 47, no. 7, pp. 536-539, 2012.

[69] K. J. Pearson, J. A. Baur, K. N. Lewis et al., "Resveratrol delays age-related deterioration and mimics transcriptional aspects of dietary restriction without extending life span," Cell Metabolism, vol. 8, no. 2, pp. 157-168, 2008.

[70] S. Zou, J. R. Carey, P. Liedo et al., "The prolongevity effect of resveratrol depends on dietary composition and calorie intake in a tephritid fruit fly," Experimental Gerontology, vol. 44, no. 6-7, pp. 472-476, 2009.

[71] C. Wang, C. T. Wheeler, T. Alberico et al., "The effect of resveratrol on lifespan depends on both gender and dietary nutrient composition in Drosophila melanogaster," Age. In press.

[72] P. Liedo, J. R. Carey, D. K. Ingram, and S. Zou, “The interplay among dietary fat, sugar, protein and acai (Euterpe oleracea Mart.) pulp in modulating lifespan and reproduction in a Tephritid fruit fly," Experimental Gerontology, vol. 47, no. 7, pp. 536-539, 2012.

[73] N. G. Baydar, H. Baydar, and T. Debener, "Analysis of genetic relationships among Rosa damascena plants grown in Turkey by using AFLP and microsatellite markers," Journal of Biotechnology, vol. 111, no. 3, pp. 263-267, 2004.

[74] T. Hongratanaworakit, "Relaxing effect of rose oil on humans," Natural Product Communications, vol. 4, no. 2, pp. 291-296, 2009.

[75] N. Kumar, P. Bhandari, B. Singh, A. P. Gupta, and V. K. Kaul, "Reversed phase-HPLC for rapid determination of polyphenols in flowers of rose species," Journal of Separation Science, vol. 31, no. 2, pp. 262-267, 2008.

[76] E. Basim and H. Basim, "Antibacterial activity of Rosa damascena essential oil,” Fitoterapia, vol. 74, no. 4, pp. 394396, 2003.

[77] R. Ramezani, A. Moghimi, H. Rakhshandeh, H. Ejtehadi, and M. Kheirabadi, "The effect of Rosa damascena essential oil on the amygdala electrical kindling seizures in rat," Pakistan Journal of Biological Sciences, vol. 11, no. 5, pp. 746-751, 2008.

[78] N. Shokouhinejad, M. Emaneini, M. Aligholi, and F. Jabalameli, "Antimicrobial effect of Rosa damascena extract on selected endodontic pathogens," Journal of the California Dental Association, vol. 38, no. 2, pp. 123-126, 2010.

[79] S. Awale, C. Tohda, Y. Tezuka et al., "Protective effects of Rosa damascena and its active constituent on abeta(25-35)induced neuritic atrophy," Evidence-based Complementary and Alternative Medicine, vol. 2011, Article ID 131042, 2011.

[80] S. E. Schriner, N. S. Katoozi, K. Q. Pham, M. Gazarian, A. Zarban, and M. Jafari, "Extension of Drosophila lifespan by Rosa damascena associated with an increased sensitivity to heat," Biogerontology, vol. 13, no. 2, pp. 105-117, 2012.

[81] J. H. Weisburger, "Chemopreventive effects of cocoa polyphenols on chronic diseases," Experimental Biology and Medicine, vol. 226, no. 10, pp. 891-897, 2001.

[82] P. Martorell, J. V. Forment, R. De Llanos et al., "Use of Saccharomyces cerevisiae and Caenorhabditis elegans as model organisms to study the effect of cocoa polyphenols in the resistance to oxidative stress," Journal of Agricultural and Food Chemistry, vol. 59, no. 5, pp. 2077-2085, 2011.

[83] N. T. Zaveri, "Green tea and its polyphenolic catechins: medicinal uses in cancer and noncancer applications," Life Sciences, vol. 78, no. 18, pp. 2073-2080, 2006. 
[84] S. M. Sagar, D. Yance, and R. K. Wong, "Natural health products that inhibit angiogenesis: a potential source for investigational new agents to treat cancer-part 1," Current Oncology, vol. 13, no. 1, pp. 14-26, 2006.

[85] J. A. Yang, J. H. Choi, and S. J. Rhee, "Effects of green tea catechin on phospholipase A2 activity and antithrombus in streptozotocin diabetic rats," Journal of Nutritional Science and Vitaminology, vol. 45, no. 3, pp. 337-346, 1999.

[86] P. G. Pietta, "Flavonoids as antioxidants," Journal of Natural Products, vol. 63, no. 7, pp. 1035-1042, 2000.

[87] S. G. Khan, S. K. Katiyar, R. Agarwal, and H. Mukhtar, "Enhancement of antioxidant and phase II enzymes by oral feeding of green tea polyphenols in drinking water to SKH1 hairless mice: possible role in cancer chemoprevention," Cancer Research, vol. 52, no. 14, pp. 4050-4052, 1992.

[88] Y. M. Li, H. Y. E. Chan, Y. Huang, and Z. Y. Chen, "Green tea catechins upregulate superoxide dismutase and catalase in fruit flies," Molecular Nutrition and Food Research, vol. 51, no. 5, pp. 546-554, 2007.

[89] Y. M. Li, H. Y. E. Chan, X. Q. Yao, Y. Huang, and Z. Y. Chen, "Green tea catechins and broccoli reduce fat-induced mortality in Drosophila melanogaster," Journal of Nutritional Biochemistry, vol. 19, no. 6, pp. 376-383, 2008.

[90] S. Abbas and M. Wink, "Epigallocatechin gallate from green tea (Camellia sinensis) increases lifespan and stress resistance in Caenorhabditis elegans," Planta Medica, vol. 75, no. 3, pp. 216-221, 2009.

[91] L. Zhang, G. Jie, J. Zhang, and B. Zhao, "Significant longevity-extending effects of EGCG on Caenorhabditis elegans under stress," Free Radical Biology and Medicine, vol. 46, no. 3, pp. 414-421, 2009.

[92] K. Zarse, S. Jabin, and M. Ristow, "L: -Theanine extends lifespan of adult Caenorhabditis elegans," European Journal of Nutrition, vol. 51, no. 6, pp. 765-768, 2012.

[93] T. I. Kim, Y. K. Lee, S. G. Park et al., "l-Theanine, an amino acid in green tea, attenuates $\beta$-amyloid-induced cognitive dysfunction and neurotoxicity: reduction in oxidative damage and inactivation of ERK/p38 kinase and NF- $\kappa \mathrm{B}$ pathways," Free Radical Biology and Medicine, vol. 47, no. 11, pp. 1601-1610, 2009.

[94] J. Bryan, "Psychological effects of dietary components of tea: caffeine and L-theanine," Nutrition Reviews, vol. 66, no. 2, pp. 82-90, 2008.

[95] Y. Sadzuka, T. Sugiyama, A. Miyagishima, Y. Nozawa, and S. Hirota, "The effects of theanine, as a novel biochemical modulator, on the antitumor activity of adriamycin," Cancer Letters, vol. 105, no. 2, pp. 203-209, 1996.

[96] H. Yokogoshi, Y. Kato, Y. M. Sagesaka, T. Takihara-Matsuura, T. Kakuda, and N. Takeuchi, "Reduction effect of theanine on blood pressure and brain 5-hydroxyindoles in spontaneously hypertensive rats," Bioscience, Biotechnology, and Biochemistry, vol. 59, no. 4, pp. 615-618, 1995.

[97] G. Buckland, A. Agudo, N. Travier et al., "Adherence to the Mediterranean diet reduces mortality in the Spanish cohort of the European Prospective Investigation into Cancer and Nutrition (EPIC-Spain)," British Journal of Nutrition, vol. 106, no. 10, pp. 1581-1591, 2011.

[98] M. Gerber, "Biofactors in the Mediterranean diet," Clinical Chemistry and Laboratory Medicine, vol. 41, no. 8, pp. 9991004, 2003.

[99] P. Lagiou, D. Trichopoulos, S. Sandin et al., "Mediterranean dietary pattern and mortality among young women: a cohort study in Sweden," British Journal of Nutrition, vol. 96, no. 2, pp. 384-392, 2006.
[100] J. C. Stoclet, T. Chataigneau, M. Ndiaye et al., "Vascular protection by dietary polyphenols," European Journal of Pharmacology, vol. 500, no. 1-3, pp. 299-313, 2004.

[101] A. Trichopoulou, "Modified Mediterranean diet and survival: EPIC-elderly prospective cohort study," British Medical Journal, vol. 330, no. 7498, pp. 991-995, 2005.

[102] A. Bendini, L. Cerretani, A. Carrasco-Pancorbo et al., "Phenolic molecules in virgin olive oils: a survey of their sensory properties, health effects, antioxidant activity and analytical methods. An overview of the last decade," Molecules, vol. 12, no. 8, pp. 1679-1719, 2007.

[103] A. Cañuelo, B. Gilbert-López, P. Pacheco-Liñán, E. MartínezLara, E. Siles, and A. Miranda-Vizuete, "Tyrosol, a main phenol present in extra virgin olive oil, increases lifespan and stress resistance in Caenorhabditis elegans," Mechanisms of Ageing and Development, vol. 133, no. 8, pp. 563-574, 2012.

[104] A. L. Hsu, C. T. Murphy, and C. Kenyon, "Regulation of aging and age-related disease by DAF-16 and heat-shock factor," Science, vol. 300, no. 5622, pp. 1142-1145, 2003.

[105] A. W. Boots, G. R. M. M. Haenen, and A. Bast, "Health effects of quercetin: from antioxidant to nutraceutical," European Journal of Pharmacology, vol. 585, no. 2-3, pp. 325-337, 2008.

[106] K. Pietsch, N. Saul, R. Menzel, S. R. Stürzenbaum, and C. E. W. Steinberg, "Quercetin mediated lifespan extension in Caenorhabditis elegans is modulated by age-1, daf -2 , sek-1 and unc-43," Biogerontology, vol. 10, no. 5, pp. 565-578, 2009.

[107] N. Saul, K. Pietsch, R. Menzel, and C. E. W. Steinberg, "Quercetin-mediated longevity in Caenorhabditis elegans: is DAF-16 involved?" Mechanisms of Ageing and Development, vol. 129, no. 10, pp. 611-613, 2008.

[108] N. Saul, K. Pietsch, R. Menzel, S. R. Stürzenbaum, and C. E. W. Steinberg, "The longevity effect of tannic acid in Caenorhabditis elegans: disposable soma meets hormesis," Journals of Gerontology, vol. 65, no. 6, pp. 626-635, 2010.

[109] F. Surco-Laos, J. Cabello, E. Gómez-Orte et al., "Effects of Omethylated metabolites of quercetin on oxidative stress, thermotolerance, lifespan and bioavailability on Caenorhabditis elegans," Food \& Function, vol. 2, no. 8, pp. 445-456, 2011.

[110] A. Kampkötter, C. Timpel, R. F. Zurawski et al., "Increase of stress resistance and lifespan of Caenorhabditis elegans by quercetin," Comparative Biochemistry and Physiology Part B, vol. 149, no. 2, pp. 314-323, 2008.

[111] K. Yazawa, T. Kihara, H. Shen, Y. Shimmyo, T. Niidome, and H. Sugimoto, "Distinct mechanisms underlie distinct polyphenol-induced neuroprotection," FEBS Letters, vol. 580, no. 28-29, pp. 6623-6628, 2006.

[112] T. J. Kim, J. L. Silva, and Y. S. Jung, "Antibacterial activity of fresh and processed red muscadine juice and the role of their polar compounds on Escherichia coli O157:H7," Journal of Applied Microbiology, vol. 107, no. 2, pp. 533-539, 2009.

[113] T. Taguri, T. Tanaka, and I. Kouno, "Antimicrobial activity of 10 different plant polyphenols against bacteria causing foodborne disease," Biological and Pharmaceutical Bulletin, vol. 27, no. 12, pp. 1965-1969, 2004.

[114] M. T. Huang, R. L. Chang, and A. W. Wood, "Inhibition of the mutagenicity of bay-region diol-epoxides of polycyclic aromatic hydrocarbons by tannic acid, hydroxylated anthraquinones and hydroxylated cinnamic acid derivatives," Carcinogenesis, vol. 6, no. 2, pp. 237-242, 1985.

[115] S. Taffetani, Y. Ueno, F. Meng et al., "Tannic acid inhibits cholangiocyte proliferation after bile duct ligation via a cyclic adenosine $5^{\prime}, 3^{\prime}$-monophosphate-dependent pathway," American Journal of Pathology, vol. 166, no. 6, pp. 1671-1679, 2005. 
[116] R. G. Andrade Jr., J. S. Ginani, G. K. B. Lopes, F. Dutra, A. Alonso, and M. Hermes-Lima, "Tannic acid inhibits in vitro iron-dependent free radical formation," Biochimie, vol. 88, no. 9, pp. 1287-1296, 2006.

[117] G. K. B. Lopes, H. M. Schulman, and M. Hermes-Lima, "Polyphenol tannic acid inhibits hydroxyl radical formation from Fenton reaction by complexing ferrous ions," Biochimica et Biophysica Acta, vol. 1472, no. 1-2, pp. 142-152, 1999.

[118] K. Pietsch, N. Saul, S. C. Swain, R. Menzel, C. E. Steinberg, and S. R. Stürzenbaum, "Meta-analysis of global transcriptomics suggests that conserved genetic pathways are responsible for quercetin and tannic acid mediated longevity in $C$. elegans," Frontiers in Genetics, vol. 3, article 48, 2012.

[119] J. L. Johannes and V. L. S. Cilliers, "Characterization of the products of nonenzymic autoxidative phenolic reactions in a caffeic acid model system," Journal of Agricultural and Food Chemistry, vol. 39, no. 7, pp. 1298-1303, 1991.

[120] M. R. Al-Sereiti, K. M. Abu-Amer, and P. Sen, "Pharmacology of rosemary (Rosmarinus officinalis Linn.) and its therapeutic potentials," Indian Journal of Experimental Biology, vol. 37, no. 2, pp. 124-130, 1999.

[121] A. Sahu, N. Rawal, and M. K. Pangburn, "Inhibition of complement by covalent attachment of rosmarinic acid to activated C3b," Biochemical Pharmacology, vol. 57, no. 12, pp. 1439-1446, 1999.

[122] M. A. Kelm, M. G. Nair, G. M. Strasburg, and D. L. DeWitt, "Antioxidant and cyclooxygenase inhibitory phenolic compounds from Ocimum sanctum Linn," Phytomedicine, vol. 7, no. 1, pp. 7-13, 2000.

[123] W. Zheng and S. Y. Wang, "Antioxidant activity and phenolic compounds in selected herbs," Journal of Agricultural and Food Chemistry, vol. 49, no. 11, pp. 5165-5170, 2001.

[124] K. Pietsch, N. Saul, S. Chakrabarti, S. R. Stürzenbaum, R. Menzel, and C. E. W. Steinberg, "Hormetins, antioxidants and prooxidants: defining quercetin-, caffeic acid- and rosmarinic acid-mediated life extension in C. elegans," Biogerontology, vol. 12, no. 4, pp. 329-347, 2011.

[125] T. Eisenberg, H. Knauer, A. Schauer et al., "Induction of autophagy by spermidine promotes longevity," Nature Cell Biology, vol. 11, no. 11, pp. 1305-1314, 2009.

[126] E. Morselli, G. Mariño, M. V. Bennetzen et al., "Spermidine and resveratrol induce autophagy by distinct pathways converging on the acetylproteome," Journal of Cell Biology, vol. 192, no. 4, pp. 615-629, 2011.

[127] B. Levine and G. Kroemer, "Autophagy in the pathogenesis of disease," Cell, vol. 132, no. 1, pp. 27-42, 2008.

[128] G. Scalabrino and M. E. Ferioli, "Polyamines in mammalian aging: an oncological problem, too? A review," Mechanisms of Ageing and Development, vol. 26, no. 2-3, pp. 149-164, 1984.

[129] M. Braunstein, R. E. Sobel, C. D. Allis, B. M. Turner, and J. R. Broach, "Efficient transcriptional silencing in Saccharomyces cerevisiae requires a heterochromatin histone acetylation pattern," Molecular and Cellular Biology, vol. 16, no. 8, pp. 4349-4356, 1996.

[130] E. Ford, R. Voit, G. Liszt, C. Magin, I. Grummt, and L. Guarente, "Mammalian Sir2 homolog SIRT7 is an activator of RNA polymerase I transcription," Genes and Development, vol. 20, no. 9, pp. 1075-1080, 2006.

[131] R. Mostoslavsky, K. F. Chua, D. B. Lombard et al., "Genomic instability and aging-like phenotype in the absence of mammalian SIRT6," Cell, vol. 124, no. 2, pp. 315-329, 2006.

[132] B. B. Aggarwal and K. B. Harikumar, "Potential therapeutic effects of curcumin, the anti-inflammatory agent, against neurodegenerative, cardiovascular, pulmonary, metabolic, autoimmune and neoplastic diseases," International Journal of Biochemistry and Cell Biology, vol. 41, no. 1, pp. 40-59, 2009.

[133] B. B. Aggarwal and B. Sung, "Pharmacological basis for the role of curcumin in chronic diseases: an age-old spice with modern targets," Trends in Pharmacological Sciences, vol. 30, no. 2, pp. 85-94, 2009.

[134] S. Bengmark, "Curcumin, an atoxic antioxidant and natural $\mathrm{NF} \kappa \mathrm{B}$, cyclooxygenase-2, lipooxygenase, and inducible nitric oxide synthase inhibitor: a shield against acute and chronic diseases," Journal of Parenteral and Enteral Nutrition, vol. 30, no. 1, pp. 45-51, 2006.

[135] R. K. Maheshwari, A. K. Singh, J. Gaddipati, and R. C. Srimal, "Multiple biological activities of curcumin: a short review," Life Sciences, vol. 78, no. 18, pp. 2081-2087, 2006.

[136] H. Nishino, H. Tokuda, Y. Satomi et al., "Cancer prevention by antioxidants," BioFactors, vol. 22, no. 1-4, pp. 57-61, 2004.

[137] K. S. Lee, B. S. Lee, S. Semnani et al., "Curcumin extends life span, improves health span, and modulates the expression of age-associated aging genes in Drosophila melanogaster," Rejuvenation Research, vol. 13, no. 5, pp. 561-570, 2010.

[138] V. H. Liao, C. W. Yu, Y. J. Chu, W. H. Li, Y. C. Hsieh, and T. T. Wang, "Curcumin-mediated lifespan extension in Caenorhabditis elegans," Mechanisms of Ageing and Development, vol. 132, no. 10, pp. 480-487, 2011.

[139] R. I. Morimoto, "Proteotoxic stress and inducible chaperone networks in neurodegenerative disease and aging," Genes and Development, vol. 22, no. 11, pp. 1427-1438, 2008.

[140] R. R. Kopito and D. Ron, "Conformational disease," Nature Cell Biology, vol. 2, no. 11, pp. E207-E209, 2000.

[141] D. Eisenberg and M. Jucker, "The amyloid state of proteins in human diseases," Cell, vol. 148, no. 6, pp. 1188-1203, 2012.

[142] S. Alavez, M. C. Vantipalli, D. J. S. Zucker, I. M. Klang, and G. J. Lithgow, "Amyloid-binding compounds maintain protein homeostasis during ageing and extend lifespan," Nature, vol. 472, no. 7342, pp. 226-230, 2011.

[143] D. A. Skorupa, A. Dervisefendic, J. Zwiener, and S. D. Pletcher, "Dietary composition specifies consumption, obesity, and lifespan in Drosophila melanogaster," Aging Cell, vol. 7, no. 4, pp. 478-490, 2008.

[144] S. Zou, P. Liedo, L. Altamirano-Robles et al., "Recording lifetime behavior and movement in an invertebrate model," PLoS ONE, vol. 6, no. 4, Article ID e18151, 2011.

[145] J. R. Carey, L. G. Harshman, P. Liedo, H. G. Müller, J. L. Wang, and Z. Zhang, "Longevity-fertility trade-offs in the tephritid fruit fly, Anastrepha ludens, across dietary-restriction gradients," Aging Cell, vol. 7, no. 4, pp. 470-477, 2008.

[146] G. D. Lee, M. A. Wilson, M. Zhu et al., "Dietary deprivation extends lifespan in Caenorhabditis elegans," Aging Cell, vol. 5, no. 6, pp. 515-524, 2006.

[147] M. D. W. Piper, L. Partridge, D. Raubenheimer, and S. J. Simpson, "Dietary restriction and aging: a unifying perspective," Cell Metabolism, vol. 14, no. 2, pp. 154-160, 2011.

[148] K. J. Vinknes, S. De Vogel, A. K. Elshorbagy et al., "Dietary intake of protein is positively associated with percent body fat in middle-aged and older adults," Journal of Nutrition, vol. 141, no. 3, pp. 440-446, 2011.

[149] M. Meydani and S. T. Hasan, "Dietary polyphenols and obesity," Nutrients, vol. 2, no. 7, pp. 737-751, 2010. 


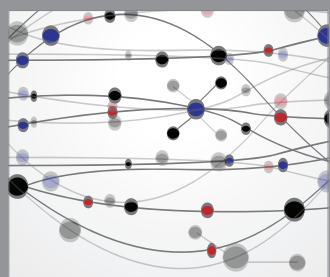

The Scientific World Journal
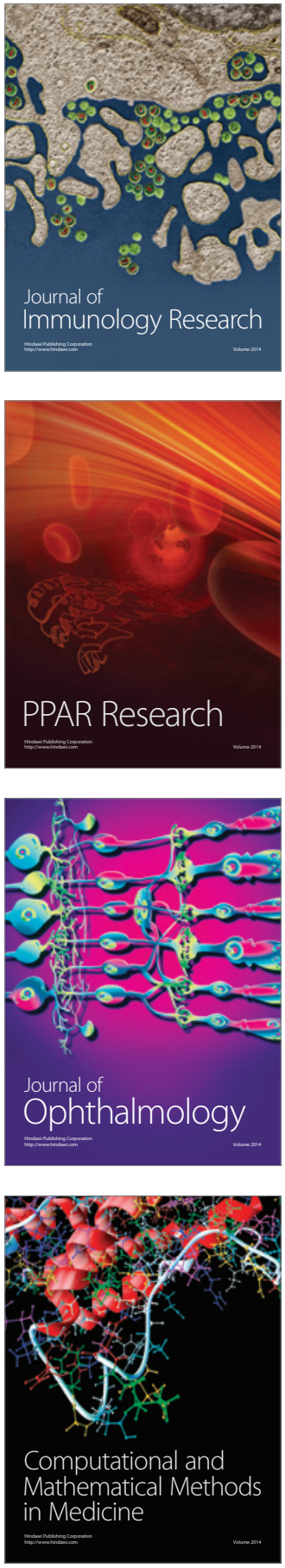

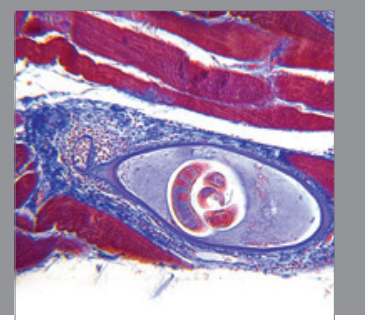

Gastroenterology

Research and Practice
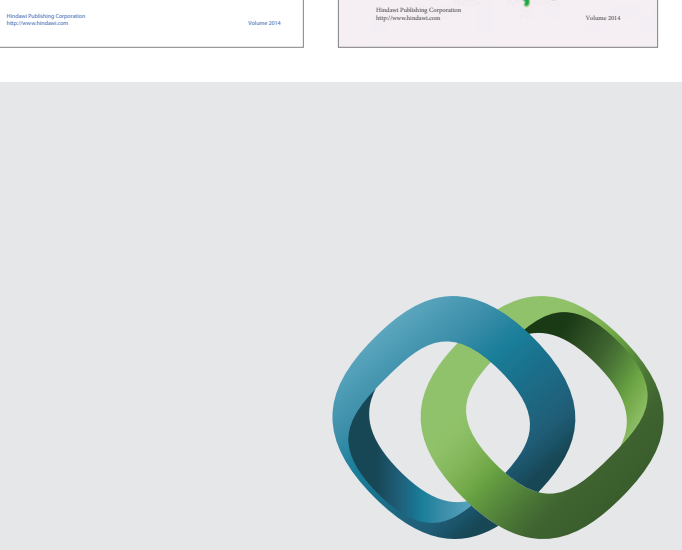

\section{Hindawi}

Submit your manuscripts at

http://www.hindawi.com
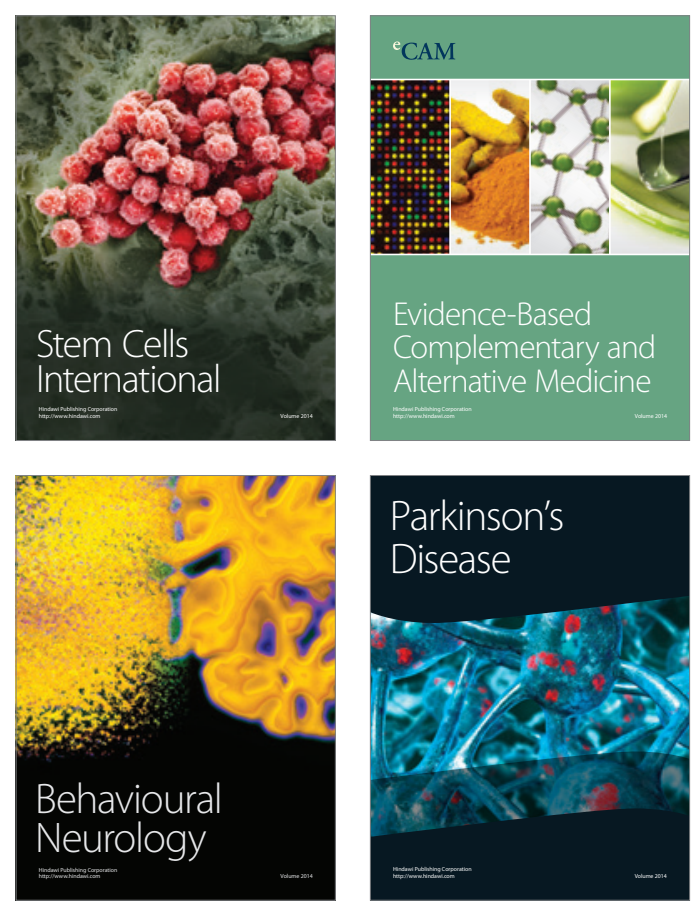

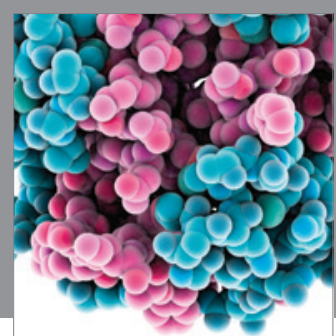

Journal of
Diabetes Research

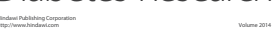

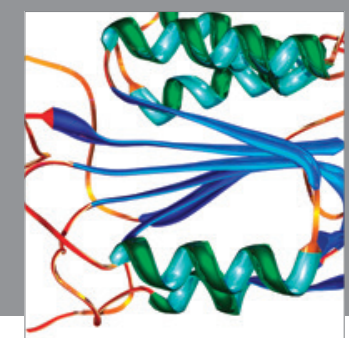

Disease Markers
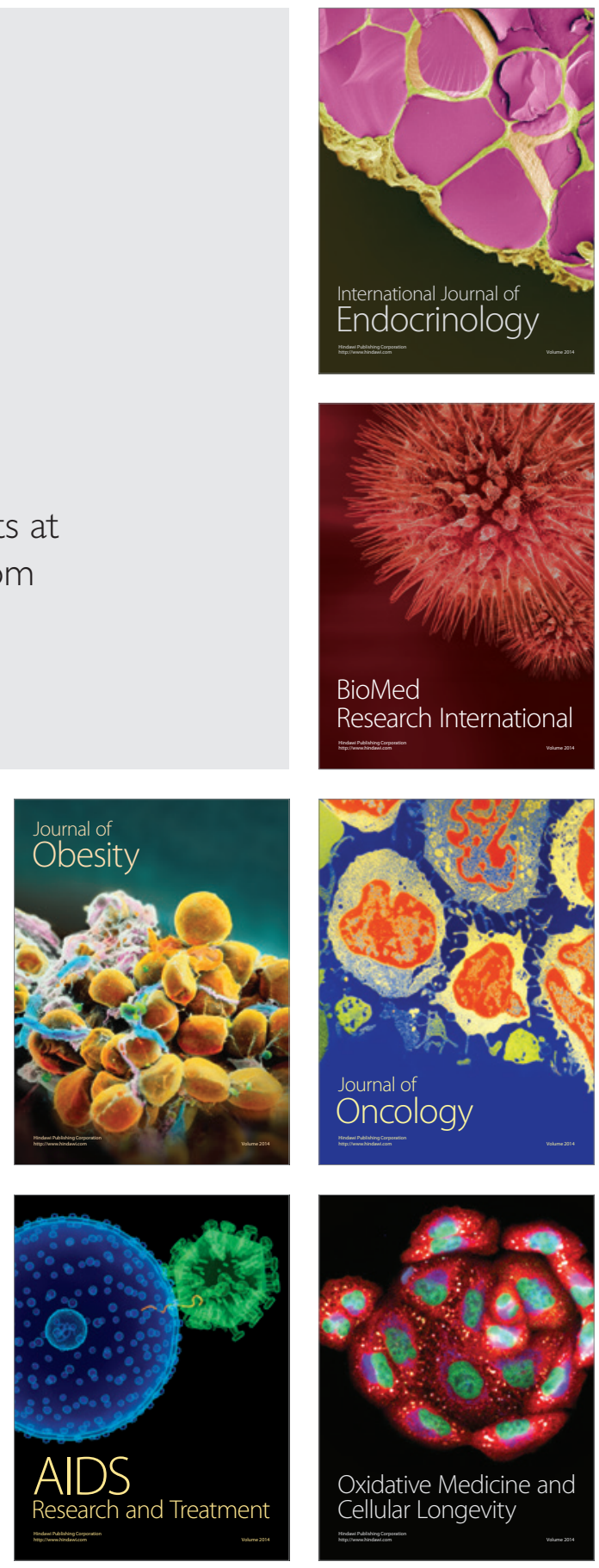